Check for updates

Cite this: RSC Adv., 2019, 9, 21646

\title{
Indium sulfide nanotubes with sulfur vacancies as an efficient photocatalyst for nitrogen fixation
}

\author{
Zhiyi He, Yu Wang, ${ }^{*}$ Xiaoli Dong, D * Nan Zheng, Hongchao Ma and Xiufang Zhang
}

We have designed and manufactured $\ln _{2} S_{3}$ nanotubes containing sulfur vacancies as effective and stable photocatalysts for nitrogen fixation and ammonia production. In the preparation process of $\ln _{2} S_{3}$, a selftemplated strategy was used to obtain the nanotubes. The sulfur vacancies were then manufactured by calcination under a nitrogen atmosphere. The existence of sulfur vacancies enhances the light absorption and promotes the separation and migration of the photoinduced charge carriers. In addition, sulfur vacancies can serve as the active sites to achieve strong $\mathrm{N}_{2}$ adsorption and activation. Thus the obtained samples show enhanced photocatalytic performance with a high $\mathrm{NH}_{3}$ generation rate $(52.49$ $\mu \mathrm{mol} \mathrm{h} \mathrm{h}^{-1} \mathrm{~g}^{-1}$ and excellent stability under UV-vis light.

Received 10th May 2019

Accepted 25th June 2019

DOI: 10.1039/c9ra03507a

rsc.li/rsc-advances recombination of photogenerated electrons and holes makes it difficult to achieve higher photocatalytic efficiency. These disadvantages make it difficult to put photocatalytic nitrogen fixation into large-scale application. In order to solve these problems, a promising method is to synthesize visible light responsive photocatalysts and improve the generation and migration rate of carriers. ${ }^{16}$

Among many materials with visible light response, indium sulfide $\left(\operatorname{In}_{2} \mathrm{~S}_{3}\right)$ has gained wide attention because of its high responsiveness, excellent photoelectric sensitivity and low toxicity. ${ }^{17-22}$ However, single-metal sulfides do not have satisfactory catalytic properties, mainly due to the separation of charge carriers, slow migration kinetics of charge carriers and low quantum efficiency. ${ }^{23,24}$ In order to solve these problems, one possible approach is to carry out surface defect engineering. ${ }^{25}$ Extensive research has been conducted on surface defect engineering, in which surface vacancy defects provide more active sites for materials and facilitate the chemical adsorption and activation of $\mathrm{N}_{2}$, thus improving the photocatalytic activity. ${ }^{26-28}$ There are many examples of sulfur vacancies and oxygen vacancies used in photocatalysts (e.g. $\mathrm{MoS}_{2}$, $\mathrm{TiO}_{2}$ ), which confirm that the presence of vacancies can significantly improve the photocatalytic properties of catalysts. After being modified, materials usually possess more holeelectron pairs, faster carrier transmission rates, and better photocatalytic performance. Therefore, it should be feasible to create sulfur vacancies in $\operatorname{In}_{2} S_{3}$ to improve its photocatalytic properties.

To improve the photocatalytic performance, another effective method is to change the morphology of the material to improve its specific surface area and to increase the contact area with the reaction medium. ${ }^{29}$ Two-dimensional hollow nanotubes with high surface area are beneficial to reduce the diffusion distance of carriers and increase the contact area with the
School of Light Industry and Chemical Engineering, Dalian Polytechnic University, \#1 Qinggongyuan, Dalian 116034, P. R. China. E-mail: wang_yu@dlpu.edu.cn; dongxiaoli65@163.com 
water medium in the reaction. In addition, the hollow space can also enhance the capture of photons by internal light scattering and reflection. ${ }^{30-33}$ As a new type of porous crystal materials, metal-organic frameworks (MOFs) are the preferred template. ${ }^{34,35}$ Because of their unique composition of metal clusters and organic bridges, they are promising templates for vulcanization in situ to obtain $\operatorname{In}_{2} \mathrm{~S}_{3}$ catalysts that retain their $2 \mathrm{D}$ morphology. At the same time, due to the loss of organic linkers in the process of solvothermal treatment, a hollow structure is formed.

In this article, $\operatorname{In}_{2} \mathrm{~S}_{3}$ hollow nanotubes were successfully prepared using MIL-68 (In) prism as a precursor via selfassembly technology. Subsequently, the as-prepared $\operatorname{In}_{2} \mathrm{~S}_{3}$ nanotubes were annealed in a nitrogen atmosphere under different temperatures to form sulfur vacancies. The nitrogen fixation performance of the obtained samples was determined using deionized water as the medium under mild reaction conditions. The crystal structure, morphology, chemical element composition, optical and electrochemical properties of the as-obtained sulfide-vacancy-decorated $\operatorname{In}_{2} \mathrm{~S}_{3}$ nanotubes were investigated. In the presence of sulfur vacancies, the photocatalytic properties show a significant improvement. Therefore, this work may indicate new directions and useful insights for the design of metal sulfide photocatalysts and their application for nitrogen fixation.

\section{Experimental}

\section{Materials and reagents}

Indium nitrate $\left(\mathrm{In}\left(\mathrm{NO}_{3}\right)_{3} \cdot x \mathrm{H}_{2} \mathrm{O}\right)$ and 1,4-benzenedicarboxylic acid $\left(\mathrm{H}_{2} \mathrm{BDC}\right)$ were supplied by Aladdin Reagent Co., Ltd. (Shanghai, China). Thiourea was obtained from Tianjin Bodi Chemical Co., Ltd. N,N-Dimethylformamide (DMF) was supplied by Sinopharm Chemical Reagent Co., Ltd. (Shanghai, China), and absolute ethanol (EtOH, 99.7\%) was obtained from Tianli Chemical Reagent Co., Ltd. (Tianjin, China). All reagents and solvents were used as received without further purification.

Synthesis of MIL-68 (In) prisms. The MIL-68 (In) hexagonal prisms were synthesized according to a reported method with some modifications. ${ }^{36}$ Firstly, $0.408 \mathrm{~g}$ of $\mathrm{In}\left(\mathrm{NO}_{3}\right)_{3} \cdot x \mathrm{H}_{2} \mathrm{O}$ and $0.2 \mathrm{~g}$ of $\mathrm{H}_{2} \mathrm{BDC}$ were dissolved in $12 \mathrm{~mL}$ DMF and stirred for $20 \mathrm{~min}$. The resultant mixture was then transferred into a Teflon-lined autoclave, which was sealed and heated at $100{ }^{\circ} \mathrm{C}$ for $48 \mathrm{~h}$. After the reaction, the white precipitate was filtrated and washed with DMF and ethanol several times, and dried in vacuum at $60{ }^{\circ} \mathrm{C}$ for $12 \mathrm{~h}$.

Synthesis of $\operatorname{In}_{2} S_{3}$ nanotubes. The $\operatorname{In}_{2} S_{3}$ nanotubes were synthesized according to a modification of a previously reported solvothermal method. ${ }^{37}$ In brief, $0.6 \mathrm{~g}$ of thiourea was initially dissolved in $80 \mathrm{~mL}$ of ethanol. Subsequently, $0.012 \mathrm{~g}$ of MIL-68 (In) was added into the solution with stirring. The obtained mixture was transferred into a Teflon-lined autoclave, sealed and heated at $180{ }^{\circ} \mathrm{C}$ for $3 \mathrm{~h}$. After cooling to room temperature, the yellow precipitate was filtrated and washed with water and ethanol four times, and finally dried at $60{ }^{\circ} \mathrm{C}$.

Synthesis of $\mathbf{S V}-\mathrm{In}_{2} \mathbf{S}_{3}$ nanotubes. The sulfur vacancycontaining (SV)- $\operatorname{In}_{2} \mathrm{~S}_{3}$ nanotubes were prepared through a simple annealing process from $\operatorname{In}_{2} \mathrm{~S}_{3}$ nanotubes. A moderate amount of $\operatorname{In}_{2} S_{3}$ nanotubes was loaded in an alumina boat and placed in a tube furnace, and the furnace was heated to $200{ }^{\circ} \mathrm{C}$ or $300{ }^{\circ} \mathrm{C}$ with a heating rate of $3{ }^{\circ} \mathrm{C} \mathrm{min}^{-1}$ and maintained for $2 \mathrm{~h}$ in nitrogen atmosphere. The materials obtained were designated as SV-In $\mathrm{S}_{3}-200$ and $\mathrm{SV}-\mathrm{In}_{2} \mathrm{~S}_{3}-300$, respectively.

\section{Materials characterization}

The transmission electron microscopy (TEM) analyses were performed by a JEOL JEM-2100F transmission electron microscope. Scanning electron microscopy (SEM) images were taken using a field emission scanning electron microscope (JSM7800F, JEOL). The crystallinity and the purity of the asprepared samples were characterized by powder X-ray diffraction (XRD) analysis on a Shimadzu XRD-6100 diffractometer at $40 \mathrm{kV}$ and $40 \mathrm{~mA}$ with $\mathrm{Cu} \mathrm{K} \alpha$ radiation. Light absorption properties were evaluated by UV-vis diffuse reflectance spectroscopy (UV-vis DRS, CARY 100\&300, VARIAN), for which $\mathrm{BaSO}_{4}$ was used as a reflectance standard. The photoluminescence (PL) spectra of the photocatalysts were acquired using a Hitachi F-7000 fluorescence spectrophotometer. Electron spin resonance (ESR) spectra were recorded on a JES-FA200 electron spin resonance spectrometer operating at room temperature. Nitrogen temperature-programmed desorption experiments $\left(\mathrm{N}_{2}\right.$-TPD) were performed by a NOVA 2000e Surface Area \& Pore Size Analyzer. X-ray photoelectron spectroscopy (XPS) spectra were conducted on a VG Scientific ESCALAB 250 XPS instrument.

\section{Photocatalytic activity measurements}

All the photocatalytic $\mathrm{N}_{2}$ fixation experiments were conducted in a sealed quartz reactor under UV-vis light irradiation by a $300 \mathrm{~W}$ Xe lamp (PLS-SXE 300, Beijing Perfect Light Co., Ltd.). Typically, 20 mg of catalysts was dispersed in $100 \mathrm{~mL}$ of ultrapure water in a quartz reactor, which was equipped with a water circulator. The mixture solution was stirred in the dark with pure $\mathrm{N}_{2}$ bubbled at a flow rate of $80 \mathrm{~mL} \mathrm{~min}^{-1}$ for $30 \mathrm{~min}$ to reach adsorption equilibrium. In the reaction process, $5 \mathrm{~mL}$ of reaction solution was taken out every $30 \mathrm{~min}$ and the catalyst was further removed through centrifugation. Throughout this reaction, the temperature of the reaction system was kept at $15{ }^{\circ} \mathrm{C}$. The concentration of product was detected using the Nessler's reagent method at $420 \mathrm{~nm}$ with an optical power meter (SDPTOP, UV2800S).

\section{Electrode preparation and electrochemical characterization}

Electrochemical and photochemical measurements of the samples were performed with a standard three-electrode setup (CHI760E Instruments). For working electrode modification, $5 \mathrm{mg}$ catalyst and $10 \mu \mathrm{L}$ Nafion solution (5 wt\%) were dispersed in a $3 \mathrm{~mL}$ volume ratio of $1: 3$ water-ethanol solution. After 20 minutes of ultrasonication, a $5 \mu \mathrm{L}$ aliquot of the mixture solution was pipetted onto the glassy carbon electrode to achieve catalyst loading, and finally dried at room temperature. Before the measurements, the $\mathrm{Na}_{2} \mathrm{SO}_{4}$ solution was purged with Ar gas for $30 \mathrm{~min}$ to remove the dissolved molecular oxygen, and purging was continued during the measurements. 


\section{Results and discussion}

As shown in Scheme $1, \operatorname{In}_{2} \mathrm{~S}_{3}$ nanotubes were prepared in two steps. First of all, MIL-68 (In) was prepared as a precursor via the solvothermal method using $\operatorname{In}\left(\mathrm{NO}_{3}\right)_{3} \cdot x \mathrm{H}_{2} \mathrm{O}$ and $\mathrm{H}_{2} \mathrm{BDC}$ as reactants. Afterward, through a liquid phase sulfidation process, the as-prepared sample was transformed into $\operatorname{In}_{2} \mathrm{~S}_{3}$ nanotubes with thiourea as a reactant. Finally, the $\operatorname{In}_{2} S_{3}$ nanotubes were calcined in a nitrogen atmosphere to produce sulfur vacancies.

The morphologies and structures of the prepared samples were illuminated by SEM, TEM and energy dispersive spectroscopy (EDS). As shown in Fig. 1a, the uniform MIL-68 (In) precursor was a hexagonal prism with a smooth surface, and its average length and average diameter were about 4-5 $\mu \mathrm{m}$ and 2 $\mu \mathrm{m}$, respectively. After sulfidation by the solvothermal method, the synthesized $\operatorname{In}_{2} S_{3}$ material perfectly maintained its $1 \mathrm{D}$ morphology with ultrathin nanosheets evenly distributed on the surface (Fig. 1b and c). TEM images show that the hexagonal prism was transformed into a hollow structure with a shell thickness of $100 \mathrm{~nm}$ after the solvothermal reaction (Fig. 1d-f). After calcination at various temperatures, no significant changes were found in the morphology and size of the samples through SEM and TEM imaging.

Elemental mappings of an individual SV- $\operatorname{In}_{2} \mathrm{~S}_{3}-200$ nanotube show a uniform distribution of $S$ and In elements across the whole nanostructure. EDS analysis reveals that the $\operatorname{In}_{2} \mathrm{~S}_{3}$ sample possesses an In/S molar ratio of $1: 1.3$. In comparison, the In/S molar ratio of $\mathrm{SV}-\mathrm{In}_{2} \mathrm{~S}_{3}-200$ is $1: 1.17$, which can be attributed to the escape of sulfur atoms.

The XRD patterns of MIL-68 (In), $\mathrm{In}_{2} \mathrm{~S}_{3}, \mathrm{SV}-\mathrm{In}_{2} \mathrm{~S}_{3}-200$ and SV$\mathrm{In}_{2} \mathrm{~S}_{3}-300$ are shown in Fig. 2. First, MIL-68 (In) was synthesized

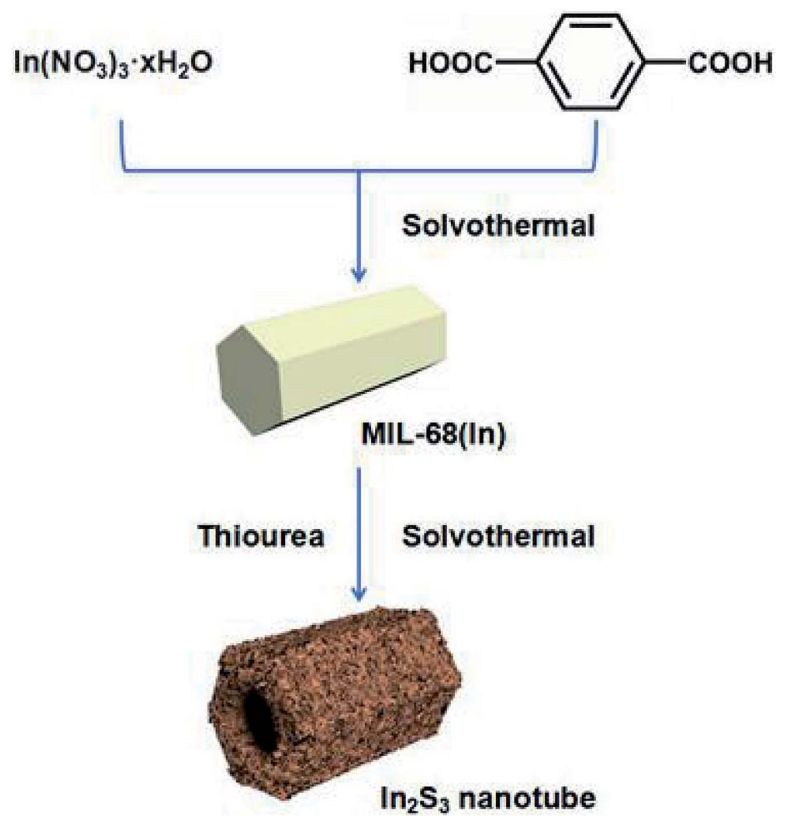

Scheme 1 A schematic illustration of the synthetic process for MIL-68 (In) and $\ln _{2} S_{3}$ nanotubes.

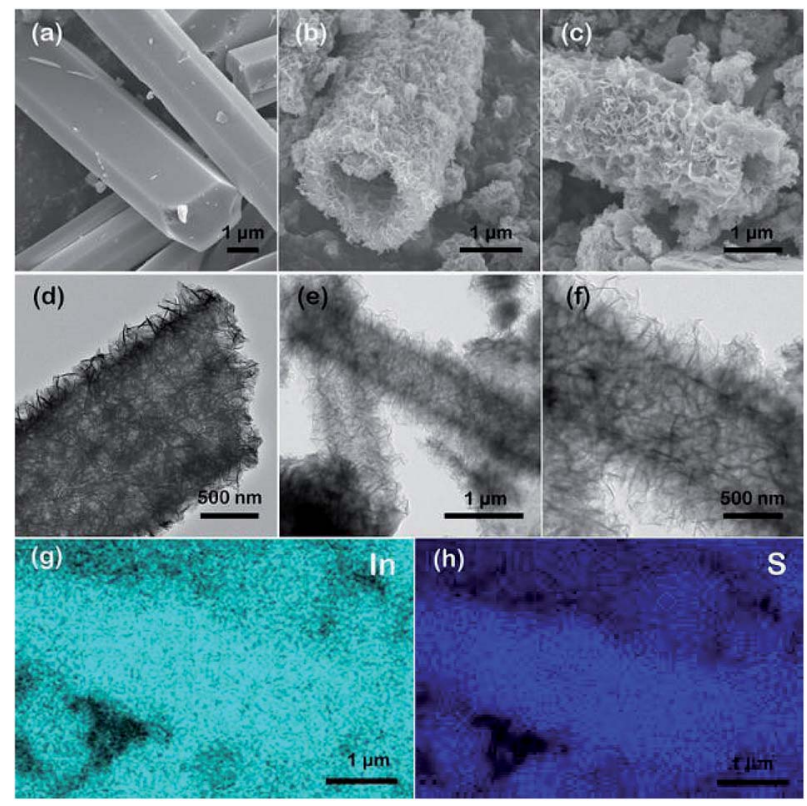

Fig. 1 SEM images of (a) MIL-68 hexagonal prisms, (b) $\ln _{2} \mathrm{~S}_{3}$ nanotubes, and (c) SV- $\ln _{2} S_{3}-200$ nanotubes, TEM images of (d) $\ln _{2} S_{3}$ nanotubes, and (e and f) SV- $\ln _{2} \mathrm{~S}_{3}-200$ nanotubes, and ( $g$ and $h$ ) elemental mapping of an individual SV- $\ln _{2} S_{3}-200$ nanotube.

as a precursor, and its successful synthesis was confirmed by XRD analysis as shown in Fig. 2a. ${ }^{38}$ The XRD patterns of $\operatorname{In}_{2} S_{3}$, SV-In ${ }_{2} \mathrm{~S}_{3}-200$ and SV-In ${ }_{2} \mathrm{~S}_{3}-300$ are shown in Fig. 2b. In the XRD pattern of $\operatorname{In}_{2} \mathrm{~S}_{3}$, the diffraction peaks located at $28.7^{\circ}, 33.2^{\circ}$ and $47.7^{\circ}$ perfectly correspond to the (222), (400) and (440) planes of $\mathrm{In}_{2} \mathrm{~S}_{3}$ (well matched with JCPDS card no. 65-0459). No diffraction peaks of MIL-68 (In) were found, indicating that MIL-68 (In) was completely converted into $\operatorname{In}_{2} \mathrm{~S}_{3}$. Moreover, it can be seen that after annealing, the peaks of $\operatorname{In}_{2} \mathrm{~S}_{3}$ were not changed, and no other impurities, such as $\operatorname{In}_{2} \mathrm{O}_{3}$ or InS, were detected, indicating the phase purity of the sample. It is worth noting that the peak positions of the samples did not change after calcination, but the strength of the peaks was reduced, which is due to the production of sulfur vacancies.

The optical properties of the samples were also evaluated by DRS. As shown in Fig. 3a, $\operatorname{In}_{2} S_{3}$ responded to visible light, with an absorption edge at about $587 \mathrm{~nm}$. Compared to pure $\operatorname{In}_{2} \mathrm{~S}_{3}$, SV-In ${ }_{2} \mathrm{~S}_{3}-200$ and $S V-\operatorname{In}_{2} \mathrm{~S}_{3}-300$ exhibit enhanced absorption in
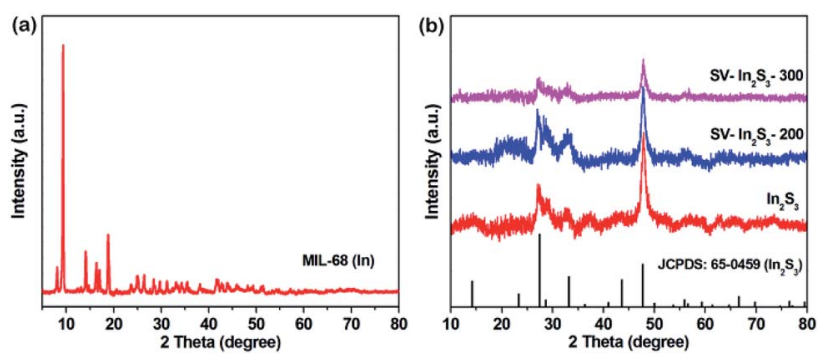

Fig. 2 XRD patterns of (a) MIL-68 (In), and (b) $\ln _{2} S_{3}$, SV- $\ln _{2} S_{3}-200$ and $S V-\ln _{2} S_{3}-300$ 
the visible light region. The absorption edge values of the SV$\mathrm{In}_{2} \mathrm{~S}_{3}-200$ and SV-In $\mathrm{S}_{3}-300$ are $590 \mathrm{~nm}$ and $608 \mathrm{~nm}$, respectively. The absorption intensity is proportional to the calcination temperature in nitrogen, indicating an increase in the number of sulfur vacancies with increasing calcination temperature. The estimated band gap energies of $\operatorname{In}_{2} \mathrm{~S}_{3}$, SV$\mathrm{In}_{2} \mathrm{~S}_{3}-200$ and $\mathrm{SV}-\mathrm{In}_{2} \mathrm{~S}_{3}-300$ are 2.17, 2.14 and $2.10 \mathrm{eV}$, respectively, corresponding to the Tauc plots shown in Fig. $3 \mathrm{~b}$.

In order to further investigate the mechanism of photocatalytic activity, the relative band positions of these materials were calculated, because the edge positions play key roles in determining the transmission of photoinduced charge carriers. The X-ray photoelectron spectroscopy (XPS) VB spectra (Fig. 3c) show that the valence bands (VB) of $\operatorname{In}_{2} S_{3}$ and $S V-\operatorname{In}_{2} S_{3}-200$ are $1.31 \mathrm{eV}$ and $1.27 \mathrm{eV}$, respectively. Furthermore, the conduction bands (CB) of the materials were determined from the MottSchottky plots (Fig. 3d). The corresponding flat band potentials of the $\mathrm{In}_{2} \mathrm{~S}_{3}$ and $\mathrm{SV}-\mathrm{In}_{2} \mathrm{~S}_{3}-200$ samples are estimated to be $-1.05 \mathrm{~V}$ and $-1.03 \mathrm{~V}$ versus $\mathrm{Ag} / \mathrm{AgCl}$, respectively. If the obtained flat band potential values are converted to normal hydrogen electrode (NHE) potentials, the values are equal to $-0.85 \mathrm{~V}$ and $-0.83 \mathrm{~V}$ versus the NHE. The $\mathrm{CB}$ potential $\left(E_{\mathrm{CB}}\right)$ is very close to the flat band potential for n-type semiconductors. Thus, the $E_{\mathrm{CB}}$ values are $-0.85 \mathrm{~V}$ and $-0.83 \mathrm{~V}$ vs. NHE for the $\operatorname{In}_{2} \mathrm{~S}_{3}$ and SV$\mathrm{In}_{2} \mathrm{~S}_{3}-200$.

Meanwhile, to further study the surface composition and chemical state of the samples, XPS measurements were carried out. As shown in Fig. 4a, the XPS spectra of the $\operatorname{In}_{2} \mathrm{~S}_{3}$ and $\mathrm{SV}-\mathrm{In}_{2} \mathrm{~S}_{3}-200$ samples are consistent with the previously reported $\operatorname{In}_{2} \mathrm{~S}_{3}$ spectra. The only elements for which peaks were detected were In, S, O and $\mathrm{C}$. The peaks of $\mathrm{C}$ and $\mathrm{O}$ possibly arose from the adsorption of gas molecules and partial oxidation of the sample due to their exposure to the atmosphere. High-resolution XPS spectra of In $3 d$ for $\operatorname{In}_{2} S_{3}$ and SV-In ${ }_{2} \mathrm{~S}_{3}-200$ samples are shown in Fig. $4 \mathrm{~b}$. For the $\operatorname{In}_{2} \mathrm{~S}_{3}$, the two strong peaks at $444.5 \mathrm{eV}$ and $452.1 \mathrm{eV}$ are attributed to bonding energies of In $3 d_{5 / 2}$ and In $3 d_{3 / 2}$, respectively. These results indicated
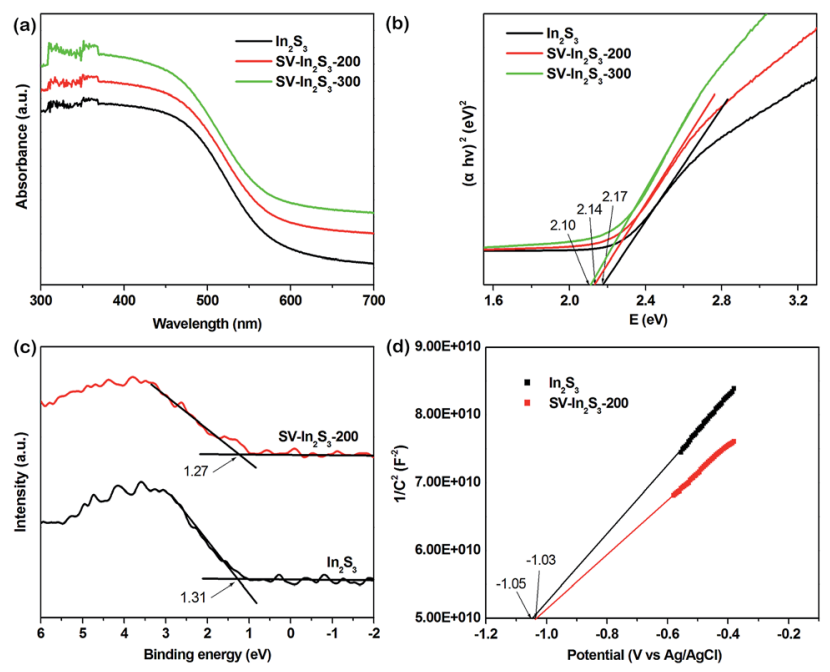

Fig. 3 (a) UV-vis diffuse reflectance spectra and (b) Tauc plots of $\ln _{2} S_{3}$, $S V-\ln _{2} S_{3}-200$ and $S V-\ln _{2} S_{3}-300$, and (c) XPS VB spectra and (d) MottSchottky plots of $\ln _{2} S_{3}$ and SV- $\ln _{2} S_{3}-200$.
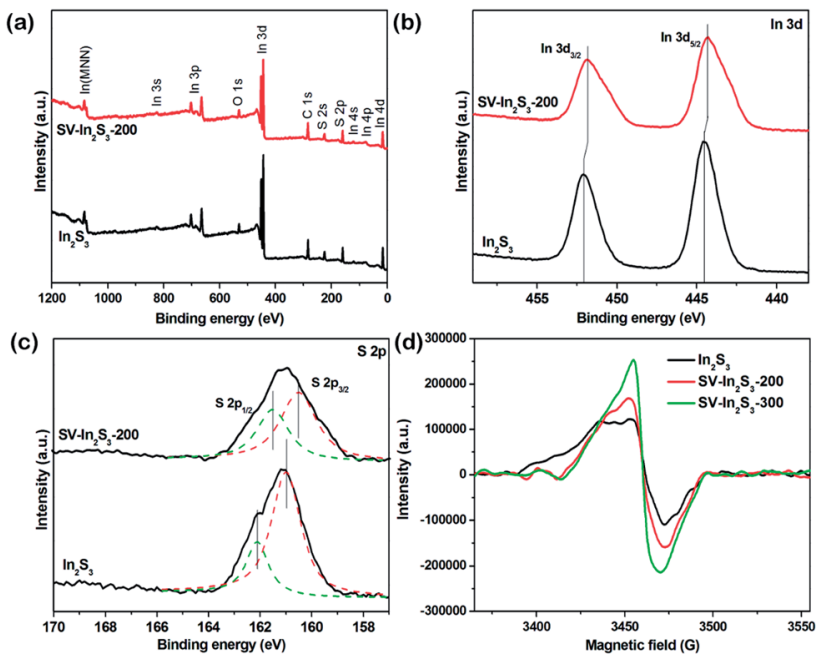

Fig. 4 (a) XPS survey spectra, and (b) In $3 d$ and (c) $S 2 p$ XPS spectra of $\ln _{2} S_{3}$ and $S V-\ln _{2} S_{3}-200$ and (d) ESR spectra of $\ln _{2} S_{3}, S V-\ln _{2} S_{3}-200$ and $S V-\ln _{2} S_{3}-300$.

that the valence state of In was +3 . Meanwhile, for the SV- $\operatorname{In}_{2} \mathrm{~S}_{3}-200$ the two peaks shifted to lower binding energies, i.e. $444.3 \mathrm{eV}$ for In $3 d_{5 / 2}$ and $451.8 \mathrm{eV}$ for In $3 d_{3 / 2}$. Fig. $4 \mathrm{c}$ shows the $\mathrm{S} 2 \mathrm{p}$ spectra of $\operatorname{In}_{2} \mathrm{~S}_{3}$ and SV-In $\mathrm{S}_{3}-200$ samples. For the $\mathrm{In}_{2} \mathrm{~S}_{3}$, two peaks at $161.1 \mathrm{eV}$ and $162.1 \mathrm{eV}$ can be assigned to the bonding energy of $S 2 p_{3 / 2}$ and $S 2 p_{1 /}$ 2. Meanwhile, for the $S V-\operatorname{In}_{2} S_{3}-200$, the peak positions shifted to lower binding energies, demonstrating the existence of sulfur vacancies. The combined energy values of the detected In $3 \mathrm{~d}$ and $\mathrm{S}$ $2 \mathrm{p}$ are consistent with the previously reported $\operatorname{In}_{2} \mathrm{~S}_{3}$ data.

ESR spectroscopy is considered as a powerful tool to probe the presence and the concentration of vacancies. ${ }^{39}$ As shown in the ESR spectra (Fig. 4d), the two calcined samples display stronger ESR signals than the original $\operatorname{In}_{2} \mathrm{~S}_{3}$, wherein the SV$\mathrm{In}_{2} \mathrm{~S}_{3}-300$ sample exhibits the highest peak among all three samples, suggesting the greatest number of unpaired electrons. This indicated that the amount of sulfur atoms in the material decreased when the calcination temperature was increased. Vacancies not only improve the photoabsorption ability of semiconductors, but also act as traps for photogenerated electrons to constrain the recombination of electron/hole pairs, thereby enhancing the photoreactivity.

The $\mathrm{N}_{2}$ adsorption-desorption measurements revealed that the $\mathrm{In}_{2} \mathrm{~S}_{3}$ possessed a high BET surface area of $110.42 \mathrm{~m}^{2} \mathrm{~g}^{-1}$, while the SV-In $\mathrm{S}_{2} \mathrm{~S}_{3}-200$ nanotubes had a BET surface area of $82.97 \mathrm{~m}^{2} \mathrm{~g}^{-1}$ (Fig. 5a). Thus, after calcination of indium sulfide, the specific surface area decreased to a certain extent. This can be attributed to the partial collapse of the structure during the calcination process, after which the material structure was no longer completely hollow. The $\mathrm{N}_{2}$ sorption isotherm shows a hysteresis loop, indicating the existence of mesopores in the structure. This mesoporous characteristic is beneficial to the adsorption of nitrogen in the photocatalytic nitrogen fixation reaction. Moreover, the presence of mesopores in the catalyst can provide more active sites for the reaction and accelerate charge transfer to promote the occurrence of photocatalytic reactions. 

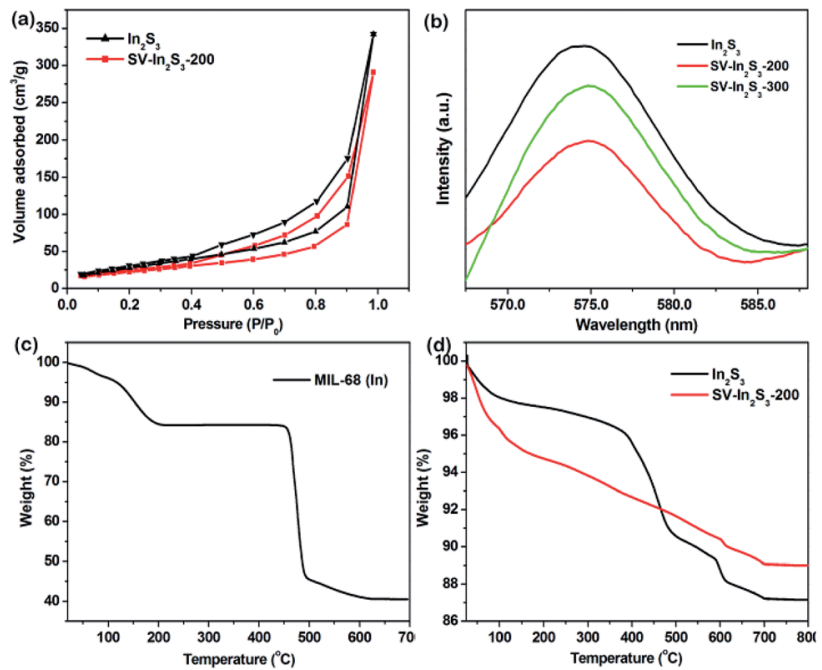

Fig. 5 (a) $N_{2}$ adsorption isotherms of $\ln _{2} S_{3}$ and $S V-\ln _{2} S_{3}-200$, (b) PL spectra of $\ln _{2} S_{3}$, SV- $\ln _{2} S_{3}-200$ and $S V-\ln _{2} S_{3}-300$, (c) TGA curve of MIL-68 (In), and (d) TGA curves of $\ln _{2} S_{3}$ and SV- $\ln _{2} S_{3}-200$.

The separation-recombination rate of photogenerated charge carriers in these materials was estimated by PL characterization (Fig. 5b). The results demonstrated that the SV- $\mathrm{In}_{2} \mathrm{~S}_{3}$ 200 sample exhibited a remarkable fluorescence quenching compared with the $\operatorname{In}_{2} S_{3}$ and SV-In ${ }_{2} S_{3}-300$ samples. This result confirms that electron-hole recombination in materials containing the appropriate amount of vacancies is greatly inhibited, which is beneficial to the photocatalytic reaction. ${ }^{\mathbf{4 0}}$

The thermal gravimetric analysis (TGA) curves under $\mathrm{N}_{2}$ atmosphere are shown in Fig. 5c and d. For MIL-68 (In), as seen in Fig. $5 \mathrm{c}$, there is a weight loss before $200{ }^{\circ} \mathrm{C}$, which can be attributed to the loss of the guest molecules in the micropores. Then, the MIL-68 (In) completely decomposes at $500{ }^{\circ} \mathrm{C}$. For $\mathrm{In}_{2} \mathrm{~S}_{3}$ and the SV- $\mathrm{In}_{2} \mathrm{~S}_{3}-300$, the first weight loss is attributed to the adsorbed water and solvent molecules in the sample (Fig. 5d). With the increase of temperature, the sulfur atoms in the materials escape, and the weight of the material is finally stabilized at temperatures above $700{ }^{\circ} \mathrm{C}$. This shows that the catalyst has good thermal stability and is not easily decomposed at high temperature.

In order to investigate the charge separation efficiencies of the samples, the photocurrent responses of the as-obtained samples were tested. As can be seen from Fig. $6 \mathrm{a}, \mathrm{In}_{2} \mathrm{~S}_{3}$ shows the lowest transient photocurrent responses, indicating the highest recombination rate of photogenerated electron-hole pairs in the $\operatorname{In}_{2} S_{3}$ crystal. Comparatively, SV-In $\mathrm{S}_{3}-200$ shows the strongest photocurrent densities, indicating the separation efficiency of photogenerated charges is notably strengthened. This may be because the appropriate amount of sulfur vacancies can facilitate the trapping of electrons from the CB of $\operatorname{In}_{2} S_{3}$ and result in enhanced charge separation ability. However, $\mathrm{SV}-\mathrm{In}_{2} \mathrm{~S}_{3}-300$ shows a lower photocurrent response than that of $\mathrm{SV}-\mathrm{In}_{2} \mathrm{~S}_{3}-200$, which suggests that excessive sulfur vacancies can act as recombination centers to inhibit the separation of the electron and hole pairs. On the other hand, as Fig. $6 \mathrm{~b}$ shows, the electrochemical impedance spectra (EIS) reveal
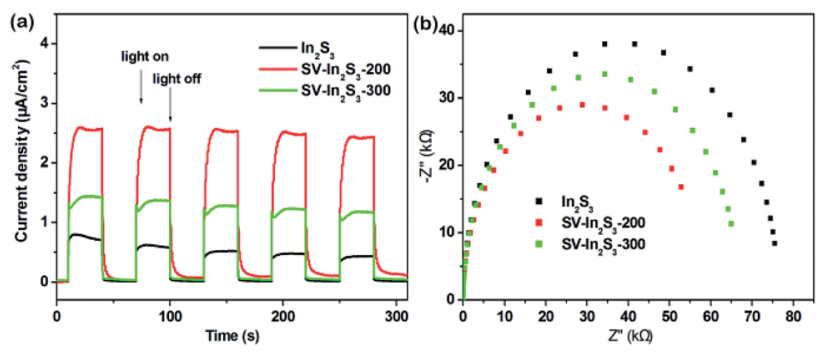

Fig. 6 (a) Transient photocurrent spectra, and (b) EIS spectra of $\ln _{2} S_{3}$, $S V-\ln _{2} S_{3}-200$ and $S V-\ln _{2} S_{3}-300$.

that $\mathrm{SV}-\mathrm{In}_{2} \mathrm{~S}_{3}-200$ manifests the smallest arc radius, demonstrating the most desirable interfacial charge transfer ability among the asobtained samples. The existence of sulfur vacancies causes a reduction in impedance, attributed to the transportation of photogenerated electron-hole pairs, which could be beneficial for enhancing the photocatalytic performance of the SV-In $\mathrm{S}_{3}-200$.

The photocatalytic nitrogen fixation properties of the $\operatorname{In}_{2} \mathrm{~S}_{3}$, SV-In ${ }_{2} \mathrm{~S}_{3}-200$ and SV- $\operatorname{In}_{2} \mathrm{~S}_{3}-300$ were tested under UV-vis light, and the results are shown in Fig. 7a. It can be seen that $\operatorname{In}_{2} S_{3}$ shows a lower nitrogen fixation ability, with the photocatalytic nitrogen fixation rate of $35.56 \mu \mathrm{mol} \mathrm{h} \mathrm{h}^{-1} \mathrm{~g}^{-1}$. However, the samples containing sulfur vacancies behave better than the original $\operatorname{In}_{2} \mathrm{~S}_{3}$, which confirmed that sulfur vacancies can improve the photocatalytic properties of materials. SV- $\operatorname{In}_{2} \mathrm{~S}_{3}-200$ exhibits a remarkable performance for nitrogen fixation of $52.49 \mu \mathrm{mol} \mathrm{h}{ }^{-1} \mathrm{~g}^{-1}$. The high nitrogen fixation rate is mainly due to an appropriate number of sulfur vacancies. In contrast, the nitrogen fixation efficiency of SV- $\mathrm{In}_{2} \mathrm{~S}_{3}-300$ is $47.41 \mu \mathrm{mol} \mathrm{h}^{-1}$ $\mathrm{g}^{-1}$. The lower $\mathrm{N}_{2}$ fixation performance of $\mathrm{SV}-\mathrm{In}_{2} \mathrm{~S}_{3}-300$ may be due to the excessive sulfur vacancies, which can act as recombination centers to inhibit the separation of the electron and hole pairs, inhibiting the photocatalytic properties. The calcination-optimized $\mathrm{SV}-\mathrm{In}_{2} \mathrm{~S}_{3}-200$ catalyst has excellent stability of nitrogen fixation and ammonia production performance under UV-vis light (Fig. 7b). After three cycles, SV-In ${ }_{2} \mathrm{~S}_{3}$ 200 still exhibits an excellent photo-nitrogen fixation rate with only a slight change. The used $\mathrm{SV}-\mathrm{In}_{2} \mathrm{~S}_{3}-200$ samples were repeatedly tested by XRD and SEM (Fig. 8), and no obvious changes were detected, indicating the stability and recyclable properties of the material. Besides, a blank experiment, shown in Fig. 7c, revealed that ammonia was not produced in the solution without light irradiation, confirming that the carrier generation is excited by light irradiation.

In the photocatalytic nitrogen fixation reaction, the sulfur vacancies can serve as the active sites to capture and activate $\mathrm{N}_{2}$ molecules. Due to the large amount of $S$ vacancies in the SV- $\operatorname{In}_{2} S_{3}$, the $\mathrm{N}_{2}$ molecules are most probably captured by these $\mathrm{S}$ vacancies and activated by donating electrons from their bonding orbitals and accepting electrons into their three antibonding orbitals. From the crystal structure in Scheme 2, it can be seen that the adsorbed $\mathrm{N}_{2}$ molecules on the $\mathrm{S}$ vacancies are most likely surrounded by In atoms. Under solar light irradiation, the photogenerated charged excitons on these In atoms may cooperate with the center-adsorbed $\mathrm{N}_{2}$ molecule and result in a possible redox 

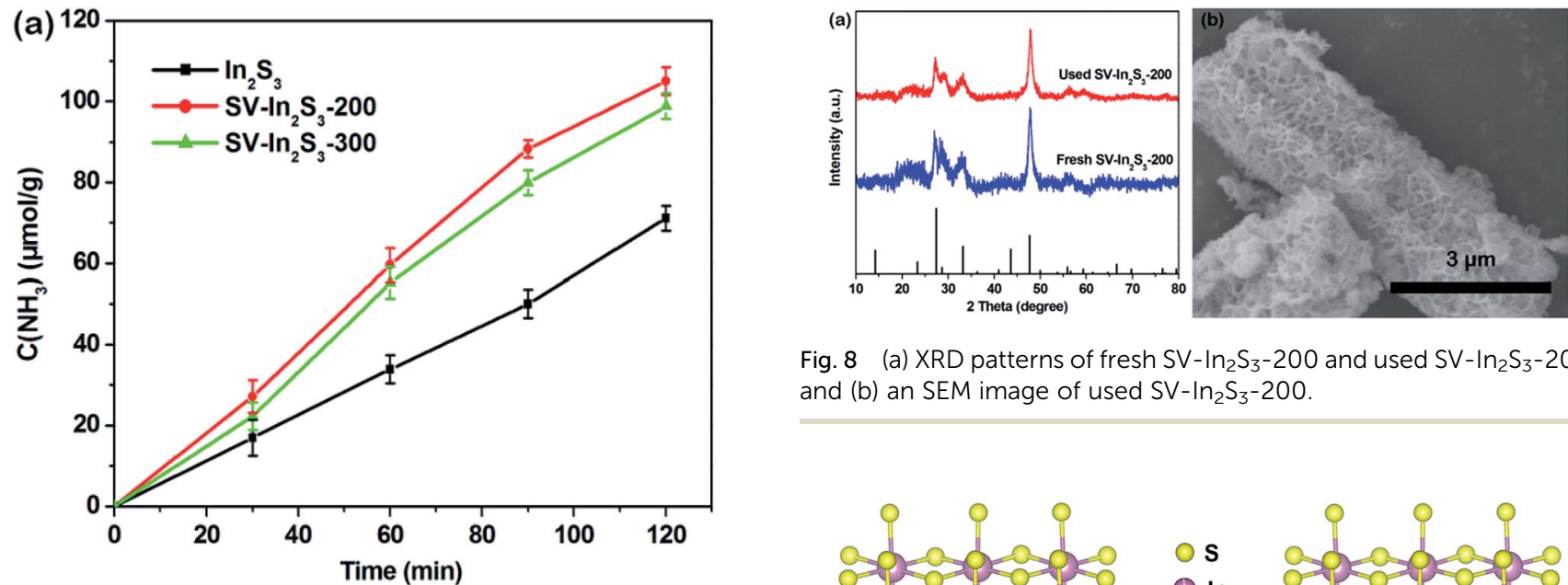

Fig. 8 (a) XRD patterns of fresh SV- $\ln _{2} S_{3}-200$ and used $S V-\ln _{2} S_{3}-200$, and (b) an SEM image of used SV- $\ln _{2} S_{3}-200$.
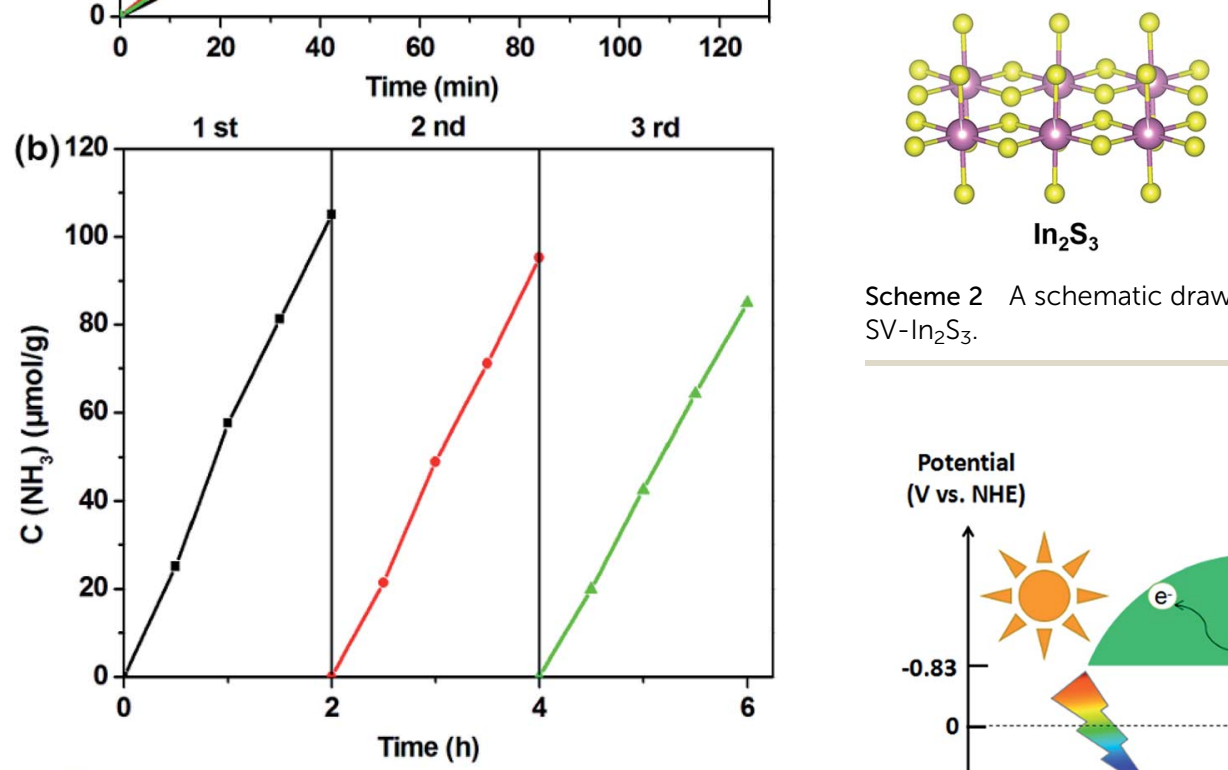

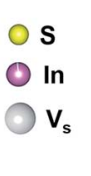

$\ln _{2} S_{3}$

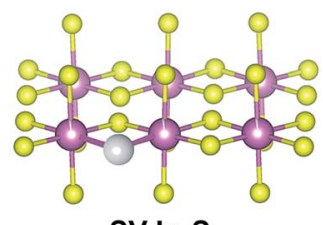

SV- $\ln _{2} S_{3}$

Scheme 2 A schematic drawing of the crystal structures of $\ln _{2} S_{3}$ and $\mathrm{SV}-\ln _{2} \mathrm{~S}_{3}$.

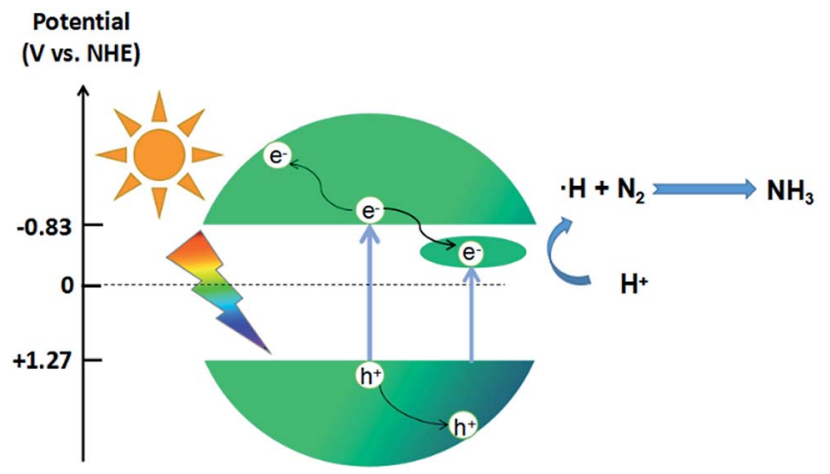

Scheme 3 The proposed photocatalytic mechanism for $\mathrm{N}_{2}$ fixation over $S V-\ln _{2} S_{3}-200$.

displays a narrower band gap and better utilization of visible light than pure $\mathrm{In}_{2} \mathrm{~S}_{3}$. Besides, $\mathrm{SV}-\mathrm{In}_{2} \mathrm{~S}_{3}-200$ is excited by light with an energy higher than the band gap, i.e., the energy needed to excite electrons from the VB to the CB or into the sulfur vacancies to form photogenerated electron-hole pairs. As active sites, sulfur vacancies accelerate the separation efficiency of photogenerated electron-hole pairs. Moreover, sulfur vacancies can also promote the adsorption and activation of $\mathrm{N}_{2}$ molecules and increase the efficiency of photocatalytic nitrogen fixation.

Fig. 7 (a) $\mathrm{N}_{2}$ fixation performance of as-prepared $\ln _{2} S_{3}, S V-\ln _{2} S_{3}-200$ and $S V-\ln _{2} S_{3}-300$, (b) cycling runs for $N_{2}$ fixation, and (c) the influence of light on the $\mathrm{N}_{2}$ fixation performance of $S V-\ln _{2} S_{3}-200$.

reaction. The mechanism of the nitrogen fixation reaction is shown in Scheme 3. Due to the existence of sulfur vacancies, an impurity energy level between the $\mathrm{CB}$ and the VB is introduced into the catalyst. Consequently, the as-obtained $\mathrm{SV}-\mathrm{In}_{2} \mathrm{~S}_{3}-200$ sample

\section{Conclusions}

In summary, $\operatorname{In}_{2} \mathrm{~S}_{3}$ hollow nanotubes were synthesized by using a self-templated strategy, and then sulfur vacancies were introduced by calcination. The obtained samples were used as an efficient and stable photocatalyst to synthesize ammonia under UV-vis light. In the process of photocatalysis, sulfur 
vacancies not only enhance the visible-light harvesting ability, but also facilitate the separation and transfer efficiency of the photogenerated electron/hole pairs. In addition, the sulfur vacancies play a key role in photocatalytic nitrogen fixation. As a result, the $\operatorname{In}_{2} \mathrm{~S}_{3}$ photocatalyst with optimized sulfur vacancies exhibits remarkable performance and outstanding stability for $\mathrm{N}_{2}$ fixation, with a stable $\mathrm{NH}_{3}$ generation rate of $52.49 \mu \mathrm{mol} \mathrm{h}^{-1}$ $\mathrm{g}^{-1}$, which is 1.48 and 1.1 times higher than those of $\operatorname{In}_{2} \mathrm{~S}_{3}$ and $\mathrm{SV}-\mathrm{In}_{2} \mathrm{~S}_{3}-300$. Besides, the unique structure and composition of these layered tubular nanostructures reduces the diffusion distance of charge carrier separation and migration, and provides a large surface area for $\mathrm{N}_{2}$ adsorption and abundant catalytically active sites for photochemical reactions. This work may provide guidelines for the design and construction of complex semiconductor-based photocatalysts for solar energyrelated applications.

\section{Conflicts of interest}

There are no conflicts to declare.

\section{Acknowledgements}

This work was financially supported by the Natural Science Foundation of China (No. 21878031), the Liaoning Province Natural Science Foundation (No. 20180550770) and the Liaoning Revitalization Talents Program (No. XLYC1802124).

\section{Notes and references}

1 V. Rosca, M. Duca, M. T. de Groot and M. T. M. Koper, Chem. Rev., 2009, 109, 2209-2244.

2 H.-P. Jia and E. A. Quadrelli, Chem. Soc. Rev., 2014, 43, 547564.

3 P. Schwerdtfeger, J. Gierlich and T. Bollwein, Angew. Chem., Int. Ed., 2003, 42, 1183.

4 N. Zhang, A. Jalil, D. Wu, S. Chen, Y. Liu, C. Gao, W. Ye, Z. Qi, H. Ju, C. Wang, X. Wu, L. Song, J. Zhu and Y. Xiong, J. Am. Chem. Soc., 2018, 140, 9434-9443.

5 B. M. Hoffman, D. Lukoyanov, Z.-Y. Yang, D. R. Dean and L. C. Seefeldt, Chem. Rev., 2014, 114, 4041-4062.

6 T. Oshikiri, K. Ueno and H. Misawa, Angew. Chem., Int. Ed., 2014, 53, 9802-9805.

7 X. Chen, N. Li, Z. Kong, W.-J. Ong and X. Zhao, Mater. Horiz., 2018, 5, 9-27.

8 J. M. P. Martirez and E. A. Carter, J. Am. Chem. Soc., 2017, 139, 4390-4398.

9 A. Kudo and Y. Miseki, Chem. Soc. Rev., 2009, 38, 253-278.

10 W.-J. Ong, L.-L. Tan, S.-P. Chai, S.-T. Yong and A. R. Mohamed, Nanoscale, 2014, 6, 1946-2008.

11 G. N. Schrauzer and T. D. Guth, J. Am. Chem. Soc., 1977, 99, 7189-7193.

12 H. Li, J. Shang, Z. Ai and L. Zhang, J. Am. Chem. Soc., 2015, 137, 6393-6399.

13 Z. Zhang, J. Huang, Y. Fang, M. Zhang, K. Liu and B. Dong, Adv. Mater., 2017, 29, 1606688.

14 A. Fujishima and K. Honda, Nature, 1972, 238, 37-38.
15 S. E. Creutz and J. C. Peters, J. Am. Chem. Soc., 2014, 136, 1105-1115.

16 W. Zhou, W. Li, J. Wang, Y. Qu, Y. Yang, Y. Xie, K. Zhang, L. Wang, H. Fu and D. Zhao, J. Am. Chem. Soc., 2014, 136, 9280-9283.

17 X. Fu, X. Wang, Z. Chen, Z. Zhang, Z. Li, D. Y. C. Leung, L. Wu and X. Fu, Appl. Catal., B, 2010, 95, 393-399.

18 W. Huang, L. Gan, H. Yang, N. Zhou, R. Wang, W. Wu, H. Li, Y. Ma, H. Zeng and T. Zhai, Adv. Funct. Mater., 2017, 27, 1702448.

19 H. Wang, Y. Wu, T. Xiao, X. Yuan, G. Zeng, W. Tu, S. Wu, H. Y. Lee, Y. Z. Tan and J. W. Chew, Appl. Catal., B, 2018, 233, 213-225.

20 H. Wang, X. Yuan, Y. Wu, G. Zeng, H. Dong, X. Chen, L. Leng, Z. Wu and L. Peng, Appl. Catal., B, 2018, 186, 19-29. 21 J. Zhang, H. Wang, X. Yuan, G. Zeng, W. Tu and S. Wang, J. Photochem. Photobiol., C, 2019, 38, 1-26.

22 Z. Wu, X. Yuan, G. Zeng, L. Jiang, H. Zhong, Y. Xie, H. Wang, X. Chen and H. Wang, Appl. Catal., B, 2018, 225, 8-21.

23 J. Zhang, H. Wang, X. Yuan, G. Zeng, W. Tu and S. Wang, J. Photochem. Photobiol. C, 2019, 38, 1-26.

24 J. Hou, S. Cao, Y. Sun, Y. Wu, F. Liang, Z. Lin and L. Sun, Adv. Energy Mater., 2018, 8, 1701114.

25 Z. Wu, X. Yuan, G. Zeng, L. Jiang, H. Zhong, Y. Xie, H. Wang, X. Chen and H. Wang, Appl. Catal., B, 2018, 225, 8-21.

26 L. Huang, J. Yang, X. Wang, J. Han, H. Han and C. Li, Phys. Chem. Chem. Phys., 2013, 15, 553-560.

27 Y. Zhao, J. Lin, Y. Liu, B. Ma, Y. Ding and M. Chen, Chem. Commun., 2015, 51, 17309-17312.

28 D. Le, T. B. Rawal and T. S. Rahman, J. Phys. Chem. C, 2014, 118, 5346-5351.

29 K. G. Kanade, J.-O. Baeg, B. B. Kale, S. Mi Lee, S.-J. Moon and K.-j. Kong, Int. J. Hydrogen Energy, 2007, 32, 4678-4684.

30 M. Zhu, Y. Osakada, S. Kim, M. Fujitsuka and T. Majima, Appl. Catal., B, 2017, 217, 285-292.

31 L. Yu, H. B. Wu and X. W. D. Lou, Acc. Chem. Res., 2017, 50, 293-301.

32 X. Lai, J. E. Halpert and D. Wang, Energy Environ. Sci., 2012, 5, 5604-5618.

33 S. Wang, B. Y. Guan, L. Yu and X. W. Lou, Adv. Mater., 2017, 29, 1702724.

34 K. B. Lausund, V. Petrovic and O. Nilsen, Dalton Trans., 2017, 46, 16983-16992.

35 H.-C. Zhou, J. R. Long and O. M. Yaghi, Chem. Rev., 2012, 112, 673-674.

36 H. Zhang, J. Nai, L. Yu and X. W. Lou, Joule, 2017, 1, 77-107. 37 C. Volkringer, M. Meddouri, T. Loiseau, N. Guillou, J. Marrot, G. Férey, M. Haouas, F. Taulelle, N. Audebrand and M. Latroche, Inorg. Chem., 2008, 47, 11892-11901.

38 S. Wang, B. Y. Guan, Y. Lu and X. W. D. Lou, J. Am. Chem. Soc., 2017, 139, 17305-17308.

39 W. Cho, H. J. Lee and M. Oh, J. Am. Chem. Soc., 2008, 130, 16943-16946.

40 X. Jiao, Z. Chen, X. Li, Y. Sun, S. Gao, W. Yan, C. Wang, Q. Zhang, Y. Lin, Y. Luo and Y. Xie, J. Am. Chem. Soc., 2017, 139, 7586-7594. 